\title{
Design and Principle of a Portable and Intelligent Bottle for Dropping Pills
}

\author{
Haihua Fu \\ Guangdong Mechanical and Electrical Polytechinc, Guangzhou, Guangdong Province, China
}

Keywords: Portable, Dropping pill, Intelligent bottle, Design, Principle

\begin{abstract}
This paper aims to design a dropping pill bottle which is portable, safe, reliable and easy to take medicine. The bottle body was made of environmental materials with the structure of a flat cuboid. On the inner wall of the bottle, an inclined drug groove was designed. The end of the drug groove was connected to a well. The control module is designed in the middle of the medicine well. The control button is extended out of the bottle body and the cap is designed as the flip cover. When taking the medicine, the dosage can be accurately controlled by using the control function of the drug groove and the control module. The dropping pill bottle is small in size, simple in structure, easy to operate and low in manufacturing cost. It is safe, hygienic, and convenient to carry and take medicine.
\end{abstract}

\section{Introduction}

At present, commonly used drug packages in the market include bag, bottle, aluminum plastic plate, tube, and so on.[1] Bottles are the most frequently used, with a market share of more than $90 \%$. Although bottles are widely used due to their low manufacturing costs, an indisputable fact is that bottles lack additional safety and adaptability required by users. [2] For example, many Chinese patent medicines are dropping pills, which are usually filled in bottles. When patients take medicine, they must pour the pills into their palms to determine the quantity. If too many pills are poured out, they must be put them back into the bottle again, which is very likely to cause the contamination of pills; the process of counting the number of pills is also time-consuming and laborious[3]. With China's entry into WTO, the country puts forward more and more strict regulations on drug management, in order to meet people's high requirements for drug packaging. Intelligent packages with advantages of environmental protection, safety, convenience, high efficiency and affordable are under exploration and development. For example, in recent years, more and more scientific research teams have joined in the research of biodegradable plastics [4]. In particular, A Kind of Intelligent Drug Taking Bottle for Dropping Pills [5] and A Kind of Variable Drug Taking Bottle Cap recently designed and patented by Haihua $\mathrm{Fu}$, have successively obtained patent certificates.

\section{Design Objectives}

Pills have the features of regular shapes and good rotary extrusion state [6]. This design starts from the needs of patients at all levels, and adheres to the intelligent design concept of environmental protection, safety, convenience, high efficiency and affordable, and the humanized design principle of people-oriented, so as to ensure the basic functions of drug bottles in protection, convenient storage and transportation, promoting drug sales, and facilitating drug information identification. [7] A portable intelligent bottle for dropping pills is designed in this paper; it is portable, easy to carry, [8] accurate in taking medicine, healthy, safe and reliable.

\section{Basic Structure and Working Principle}

\subsection{Basic Structure of the Portable Intelligent Bottle for Dropping Pills}

The portable and intelligent bottle for dropping pills is mainly composed of the bottle body, the drug guide groove, the medicine well, the control frame, the reset spring, the control button and the flip cover. 


\subsubsection{Bottle and the Guide Groove}

As shown in Figure 1, the bottle is made of environment-friendly materials and has a flat cuboid or ellipsoid structure. The wide surface is close to the bottom, and an inclined drug guide groove is arranged on the inner wall.

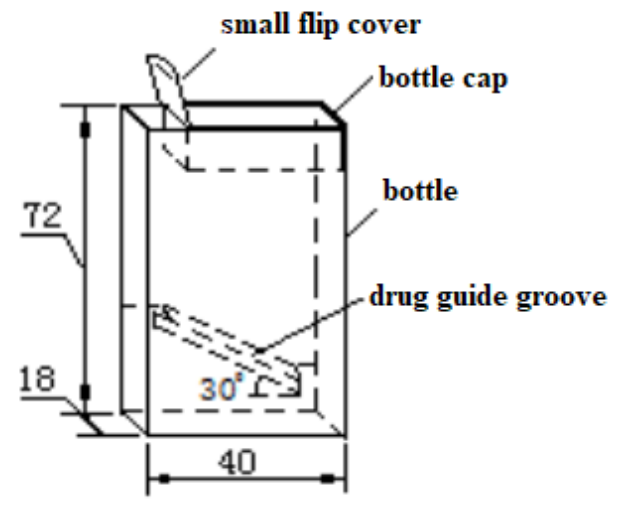

Fig.1 Bottle and the Guide Groove.

\subsubsection{Well, Frame and Spring}

As shown in Figure 2, the medicine well is a through-hole (the hole diameter is slightly larger than the diameter of the pill) made of environmental protection materials. There are slits on the walls of the hole which are perpendicular to the diameter of the pill in the middle opposite plane, which is used to install two control feet of the control frame. The function of the well is to reserve pills taken for each time. That is, before taking medicine, some pills in the bottle are stored in the well for taking.

The control frame is made of a thin aluminum (platinum) sheet after cutting, bending and pasting. The upper control foot and the lower control foot are arranged on the control frame. The vertical distance between the two feet is about the diameter of the pill. When assembling, they are respectively installed in two slits in the middle of the medicine well. Its function is to control the reserve quantity of pills at the bottom of the well (or pills below the lower control foot when the medicine bottle is inverted).

The elastic force of the spring is moderate, and it is installed between the control frame and the well. After the assembly, there is a certain pre tightening force to keep the lower control foot extending into the well under the free state. The spring is used to control the frame reset.

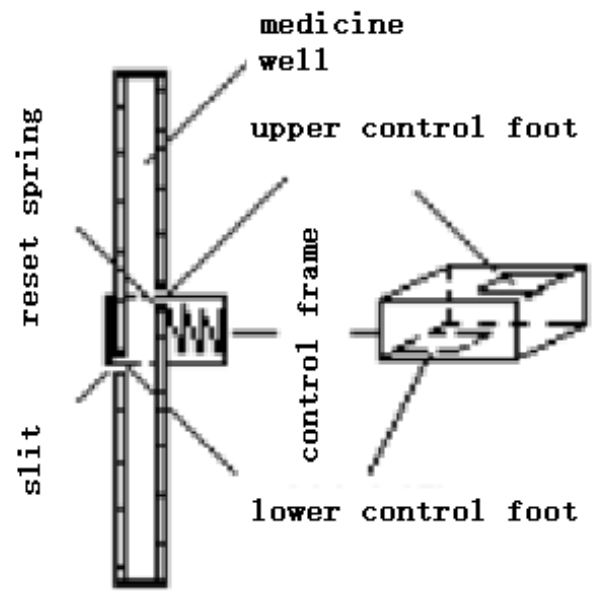

Fig.2 Well, Frame and Spring.

\subsubsection{Bottle Cap and Control Button}

The bottle cap is designed as a full flip cover or a small flip cover, which is easy to open when taking medicine and can realize fast drug taking, as shown in Figure 3. The control button is made 
of plastic, which is used to operate the control frame, as shown in Figure 4 (a).

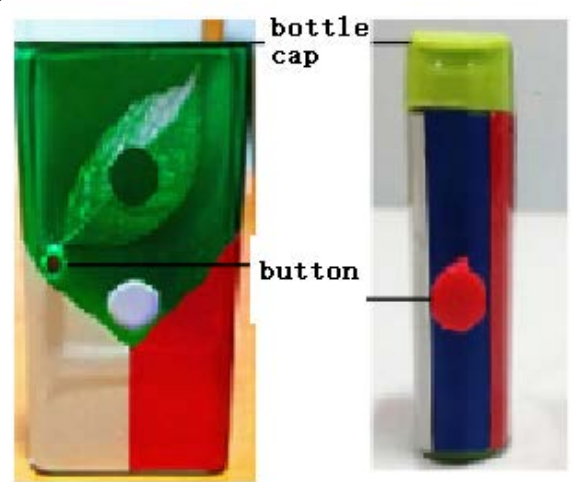
(a): flip
(b) Small flip

Fig.3 Two Portable Dropping Pill Bottles.

\subsection{Working Principle}

The drug taking process is as follows. The medicine well, the control frame and the reset spring are fixed in the inner part of the medicine bottle through the cushion block. After the medicine bottle is filled with pills, the bottle cap with the small flip cover is closed on the bottleneck in the transitional matching mode, as shown in Figure 3 (b). When taking medicine, first the patient needs to invert the medicine bottle, as shown in Figure 4 (a). Then, according to the number of pills taken by the patient at one time (usually less than 6 pills; if the patient needs more than 6 pills, he or she should take twice or several times), press the control button for corresponding numbers, and then put the bottle in the right position (as shown in Figure. 3 (b)). Finally, the patient needs to hold the medicine bottle in hand and gently open the small flip cover with the thumb; the pills can be poured in the mouth directly. The whole process can be operated by one hand with high efficiency.

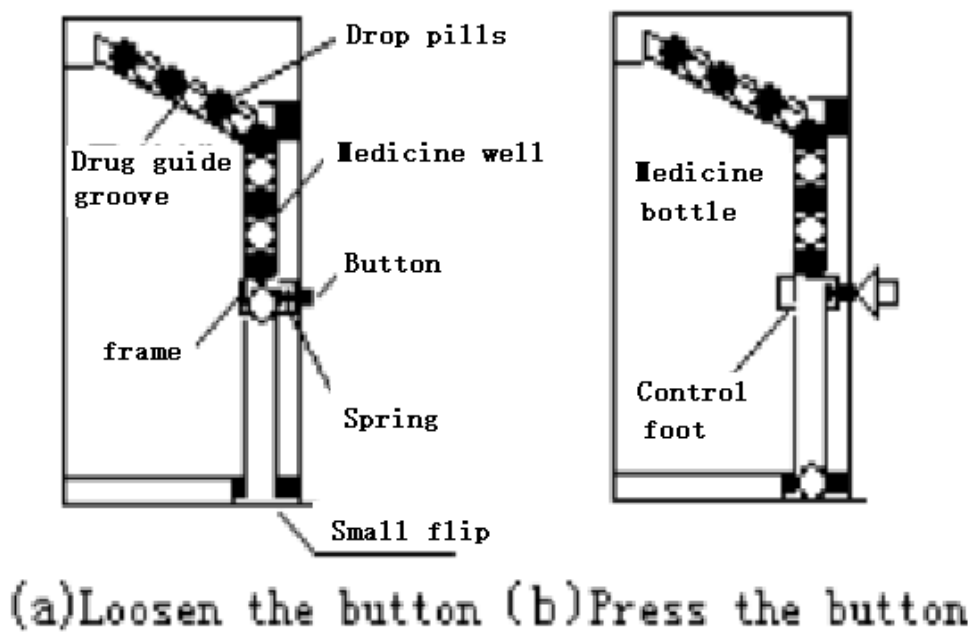

Fig.4 The Working Principle of the Bottle.

The working principle is shown in Figure 4. When the medicine bottle is inverted, under gravity and the guidance of the drug guide groove, some pills will fall into the middle of the medicine well along the guide groove, namely the upper part of the lower control foot. Because at that time, under the action of the reset spring force, the lower control foot of the control frame extends into the medicine well to stop pills from falling, as shown in Figure 4 (a). When the medicine bottle is inverted and the control button is pressed, the control frame moves to the left against the force of the reset spring, and the upper control foot of the control frame extends into the medicine well, thus blocking the second (from bottom to top) pill in the middle of the medicine well. At the same time, the lower control foot which originally blocked the first pill in the middle of the well will exit from the medicine well. Therefore, the first pill in the middle of the medicine well will fall to the bottom 
of the medicine well without any obstacles, namely above the small flip cover, as shown in Figure 4 (b). After releasing the control button, and the control frame moves to the right under the action of the reset spring force; the upper control foot of the control frame retreats out of the medicine well, and the lower control foot returns to the medicine well. When the control frame moves right to reset, pills remaining in the upper half of the medicine well also move down one pill position under gravity, as shown in Figure 3 (a). Each time you press and release the control button repeatedly, the above working process will be repeated. Pills in the upper half of the well (above the lower control foot) will be reduced, while pills in the bottom half of the well will increase until the lower half of the medicine well is stored enough pills for one time. If the dosage exceeds the maximum value for the lower half of the medicine well, the medicine can be taken twice or several times separately.

The portable intelligent bottle has following innovation points.

It is easy to carry: the shape of the bottle changes from the traditional cylinder structure to a flat cuboid or an ellipsoid structure, which is small in size and easy to carry and grasp at any time. [9] It is very suitable for work or travelling.

It is safety for children. Intelligent barrier structures, double mechanisms and specific action control in drug taking can effectively reduce safety accidents of eating and misusing dangerous goods by specific objects (children). [10]

It is intelligent and convenient. The bottle is designed to be packaged with a cap that can be opened with one finger, [11] so as to help patients take drugs more conveniently [12].

It is humanized. The humanized design of small flip and button makes it more convenient for the elderly, the blind and the disabled with one arm. It is not only simple, easy to use and easy to operate [13], but also reflects the humanistic care for some special groups.

It has wide range of application. The dosage is controlled manually by the button, which is suitable for patients of all ages. It also follows the people-oriented design concept in ergonomics, which makes things adapt to people rather than asking people to adapt to things [14].

It is highly efficient. The design can realize one hand operation, fast drug taking, labor-saving, time-saving and smooth usage. [15]

It is scientific. The accurate, scientific and humanized method of taking medicine [16] can overcome patients' bad habit of taking medicine through pouring pills into their hands. It effectively avoids the secondary pollution of drugs and even the infection of other diseases, so as to effectively protect the health and life safety of consumers [16].

It is economic [17]. The pure mechanical design and simple structure is easy to manufacture and promote. The low cost feature is also in line with the value and cultural orientation of consumers. [18]

It is expandable. In order to protect the safety of medication for the elderly and improve the selfcare ability of the elderly, the design of quantitative drug taking method can be expanded.

\section{Conclusion}

At present, the current situation of China's pharmaceutical packaging industry can be concluded as the following sentence. "Although in recent years, the scale and quantity of pharmaceutical plastic containers in China have developed rapidly, we are basically following others, and there are few patents and original inventions.” [19] The portable intelligent bottle for dropping pills has been granted a utility model patent (Patent No.: zl201721737620.9) by the National Intellectual Property Administration due to its safety, health as well as convenient carrying and drug taking. The disadvantage is that the size of drop pills should be unified and the diameter error should be within the specified range.

In a word, "the intelligent packaging full of humanization has filled in the blank with its convenient, efficient and safe performance.” [20] If it is widely used, it will greatly shorten the time for patients to take medicine, solve problems such as inconvenient drug carrying and safety accidents caused by children's ingestion of drugs by mistake, and play a significant role in improving people's quality of life. 


\section{References}

[1] Hu, F.M. The Fact and the Future of The Medicine Package Development. China Packaging Industry, vol. 12, no. 26, pp.41-44, 2002.

[2] Yi, Y. New Trend of Pharmaceutical Packaging In the Coming 10 Years in the World. China Packaging Industry, vol. 09, no. 2, pp.13, 2006.

[3] Liu, W.L. Intelligent Design of Drug Packaging Based on Safety Policy. Art \& Design, no. 11, pp. 96-97, 2015.

[4] Yang, Y.P. Research Status and Prospect of Biodegradable Plastics. Chemical Enterprise Management, no. 18, pp. 14, 2019.

[5] Xiong, M.L., Ruan, X.Y., Liu, W.L. The Strategy of Positioning the Packaging of Proprietary Chinese Medicine Based on Improving the Design Quality. Hunan Packaging, no. 6, pp. 23-26, 2018.

[6] Yue, S.L. Research and Development of Pharmaceutical Packaging Design Template, Xi'an University of Technology, 2005.

[7] Zhu, H.P., Cai, J.S., Ke, S.H. Research on Intelligent Control Drug Packaging Design. Hunan Packaging, vol. 32, no. 3, pp. 31-33, 2017.

[8] Zhao, J.R., Chang, X.Y., Wang, Y.X. Creative Design of Portable Pharmaceutical Packaging Container. Printing Technology, no. 11, pp. 24-26, 2017.

[9] Ma, H.Y., Qiu, L.B. Innovative Research on Medicine Packaging Design in China. Journal of Chuzhou University, vol. 20, no. 6, pp. 91-94, 2018.

[10] Wang, Y.Z., Ke, S.H. Packaging Design of the Obstacle Structure. Packaging Engineering, vol. 40, no. 9, pp. 107-112, 2019.

[11] Zeng, F.C., Zhang, Y.Y. Analysis and Application of Intelligent Technology in Drug Packaging Design. Design, no. 03, pp. 108-109, 2015.

[12] Yu, X.Y. Huang, S.Y. The Intelligent Future of Pharmaceutical Packaging. Printing Technology, no. 22, pp. 22-23, 2014.

[13] Ou, L. Humanized Design of Drug Packaging. Industrial Design, no. 6, pp. 110-111, 2017.

[14] Yu, Q. Humanized Design of Drug Packaging -- to Solve the Problem of Taking Medicine for the Elderly. China Packaging Industry, no. 16, pp. 29 - 31, 2013.

[15] Ren, Q.Z., He, H.H. The Study of Humanization Pharmaceutical Packaging Design. Logistics Engineering and Management,vol. 4, no. 11, pp. 151-154, 2014.

[16] Liu, W.L., Hu, S.Y. Review on the Study of Safety Design in Pharmaceutical Packaging. Hunan Packaging, no. 6, pp. 11-15, 2018.

[17] Tu, Y.F., Li, P.F. Our Export Problems Existing in the Chinese Medicine Product Packaging Design and Countermeasure Study. Guiding Journal of Traditional Chinese Medicine and Pharmacy, vol. 21, no. 13, pp. 57-59, 2015.

[18] Zhu, H.P., Yao, J. Research of the Intelligent Packaging Design: Take the Elderly Intelligent Drug Packaging as an Example. Art \& Design, no. 5, pp. 96-97, 2013.

[19] Wu, X.X. Create a Good Environment to Promote the Better and Faster Development of Plastic Containers for Medicinal Use. Shanghai Medical \& Pharmaceutical Journal, vol. 31, no. 2, pp. 7476, 2010.

[20] Wang, X.Q. Application and Development of Intelligent Packaging Design. Packaging Engineering, vol. 38, no. 16, pp. 241-243, 2017. 